\title{
Itinéraires Itinéraires
}

Littérature, textes, cultures

\section{Rejoindre une phrase, s'y poursuivre}

\section{Marielle Macé}

\section{OpenEdition}

\section{Journals}

Édition électronique

URL : http://journals.openedition.org/itineraires/2174

DOI : 10.4000/itineraires.2174

ISSN : 2427-920X

Éditeur

Pléiade

Édition imprimée

Date de publication : 1 mai 2010

Pagination : 87-98

ISBN : 978-2-296-11224-7

ISSN : $2100-1340$

Référence électronique

Marielle Macé, « Rejoindre une phrase, s'y poursuivre », Itinéraires [En ligne], 2010-1 | 2010, mis en ligne le 01 mai 2010, consulté le 03 mai 2019. URL : http://journals.openedition.org/itineraires/2174 DOI : 10.4000/itineraires.2174

\section{(c) (1) $\Theta$}

Itinéraires est mis à disposition selon les termes de la licence Creative Commons Attribution - Pas d'Utilisation Commerciale - Pas de Modification 4.0 International. 


\title{
Rejoindre une phrase, s'y poursuivre
}

\begin{abstract}
How do we live with the sentences (and not necessarily with the synthetic narratives) we read or have read? How do literary quotations become the occasion of an inner orientation in our experience? This article underlines the aesthetic movement through which we can be drawn towards authentic possibilities of ourselves in the very act of reading. To do so, it offers a dynamic vision of «style» and explores the logic of language and speech at work in Jean Paulhan's personal or theoretical writings. Paulhan considered that part of our practice of language consists in helping sentences become true and build around them new existential situations.
\end{abstract}

Keywords : reading, sentence, individualization, possibilities, Jean Paulhan Mots clés : lecture, phrase, individuation, possibles, Jean Paulhan

La passion des « possibles », le désir de « se voir autre » ont tenu toute une époque, et en ont constitué à la fois le foyer et l'énigme. Une catégorie modale s'est ainsi trouvée brusquement projetée dans l'ordre affectif ou les âges de la vie, et toute une psychologie de la littérature y a puisé ses ressources et ses aventures. Avant d'examiner la singularité et l'acuité de la pensée de Paulhan à ce sujet, j'aimerais revenir brièvement sur l'histoire de cet élan qui s'est essentiellement cristallisé autour du succès de Bergson, mais l'a aussi largement dépassé.

Dans le sillage des recherches de Théodule Ribot sur la multiplicité interne de la personnalité, ou du «bovarysme» ambivalent de Jules de Gaultier, la psychologie, la philosophie et la littérature se sont conjointement penchées sur l'élan des individus pour s'imaginer « autres ». À partir des années 1910, la catégorie mentale du " possible » a désigné ce que Thibaudet, bergsonien convaincu, appelait « un nouveau plaisir » :

La volupté suprême de la conscience consiste en une certaine possession de possibles, soit dans la jeunesse où la vie s'ouvre devant nous, soit dans la vieillesse, lorsqu'une lumière d'arrière-saison, éclairant tout ce que nous 
aurions pu être, le confond, dans le même chœur harmonieux et illusoire, avec ce que nous avons été ${ }^{1}$.

L'article que Jaques Rivière a consacré au « Roman d'aventure » en 1913 est au cœur de cette reconnaissance d'une nouvelle façon de se jeter dans la vie et d'y attendre un événement; il incarne l'attitude intellectuelle et affective à l'égard du futur et de soi qui a prévalu au sein de toute cette génération. Le plaisir qu'il décrit embrasse à la fois une idée de l'Histoire, une conception de la narrativité et une expérience psychique, c'est-à-dire une disposition de l'âme : il s'agit de sentir qu'un événement s'apprête à bondir sur vous, ou même simplement qu'il aurait pu le faire, « d'être quelqu'un à qui quelque chose arrive : Nous ne craignons rien de la minute qui va venir. Le bonheur a pris parti pour nous; il nous devance et nous attend ${ }^{2}$. » Ici le temps est déjà peuplé par les aventures prochaines : "Un événement nous épie, prêt à sauter sur nous. Tant pis! Tant mieux ${ }^{3}$. » Ce rapport euphorique à 1'Histoire et cette façon de la vivre sont évidemment une sommation pour les romanciers contemporains : l'accueil fait à l'imprévu, le goût de l'imminence s'imposent au roman et s'opposent aux plaisirs rétrogrades de la mémoire, associés par Rivière au genre de la poésie. Là où le poète symboliste était en état de mémoire, le romancier devra être « en état d'aventure ».

Bergson reviendra sur quelques malentendus dans la conception que ses contemporains se faisaient du possible, sans pour autant diminuer l'élan vital de la multiplication de soi - car cet élan vient du succès de sa philosophie et semble désormais définir l'être individuel (la « vie » bergsonienne se définit comme « une plénitude gonflée de possibilités »; le passage permanent de la multiplicité des voies à l'élection d'une seule, passage de la liberté au renoncement qui définit souverainement l'action, informe chez Bergson la tension propre à l'existence concrète; l'intensité de la vie psychique est ainsi liée à la présentation mentale d'une multiplicité virtuelle et s'identifie à l'indétermination propre à cet absolu qu'est l'élan vital). En 1920, au cours d'une conférence intitulée « Le possible et le réel », le philosophe ironise sur la tendance à concevoir les possibles comme des buts placés au terme de chemins qu'il suffirait d'emprunter, et la réalité comme la venue à l'existence de ce qui ne présentait simplement pas d'impossibilité ou d'obstacle à sa réalisation, c'est-à-dire comme l'actualisation d'une réserve finie, en attente de combinatoire et d'accroissement d'être, une « armoire

1. Albert Thibaudet, Trente ans de vie française. Le Bergsonisme, t. I, Paris, Nouvelle Revue française, 1923, p. 94. Je me permets de renvoyer à Marielle Macé, « Thibaudet et "le sens des possibles" ", Littérature, $\mathrm{n}^{\circ}$ 146, juin 2007, p. 20-35; et id. " "Nous attendons un roman qui sera"... Histoire littéraire et imminence dans le premier $\mathrm{xx}^{\mathrm{e}}$ siècle », Romanic Review, mars 2010 (à paraître).

2. Jacques Rivière, «Le roman d'aventure ", La Nouvelle Revue française, mai 1913, p. $762-763$.

3. Ibid., p. 763. 
aux possibles ${ }^{4} \gg$ dans laquelle la vie viendrait piocher ses formes. Mais Bergson n'humilie pas l'énergie d'un devenir-autre ou d'un devenir-plus; vivre selon les possibles est peut-être une illusion, l'illusion que le néant puisse jamais précéder l'être, l'illusion que nous puissions nous former au présent l'image de ce que demain sera, mais les illusions de cette sorte ont une utilité pratique, et Bergson les montre à l'œuvre dans toutes nos actions. Pour lui le mirage consiste simplement à faire de l'idée de possible une façon de se rapporter à l'avenir, en concevant nos propres ressources comme un espace de compétition entre des virtualités déjà présentes. Au contraire, le possible se crée « en arrière » à mesure que l'on produit du réel, et met en lumière la création «d'imprévisible nouveauté » que sera toujours la vie :

Au fur et à mesure que la réalité se crée, imprévisible et neuve, son image se réfléchit derrière elle dans le passé indéfini; elle se trouve ainsi avoir été, de tout temps, possible; mais c'est à ce moment précis qu'elle commence à l'avoir toujours été, et voilà pourquoi je disais que sa possibilité, qui ne précède pas sa réalité, l'aura précédée une fois la réalité apparue ${ }^{5}$.

Rivière et les possibles placés à l'horizon de l'individu, d'une conscience ivre de toucher ses potentialités et d'être en contact avec son propre futur; Bergson et les possibles rétrojectés, rendant rétrospectivement compte de tout ce dont cette conscience pouvait donc se rendre capable... Partout se joue la question du champ de capacité de l'individu, sa façon de se tenir dans un devenir absolument ouvert et de concevoir son horizon d'action. Pour l'individu moderne, il ne s'agit plus d'hériter d'un modèle auquel se conformer ou de se mesurer à un idéal bien précis, mais d'être soi-même ses propres possibles (comme le dira Sartre), de s'orienter dans le labyrinthe de sa propre multiplicité, de sentir en soi la vibration du temps vécu et sa frange d'imminence, bref de « se possibiliser » au présent. Ce nouveau plaisir a indiqué la voie aux écrivains du moment - Queneau l'a dit à sa façon :

Les années qui précédèrent la guerre firent triompher la Mode en tout domaine; c'était le temps des Ballets Russes, et du Cours de M. Bergson au Collège de France. Pour plus de précision, disons que tout cela commença l'année de la Comète, celle de 1910 bien entendu ${ }^{6} \ldots$

En 1913, l'attente et l'imagination du possible étaient cependant l'invention d'un futur qui n'aurait pas lieu; ce nouveau plaisir, celui d'être quelqu'un à qui quelque chose arrive, incarnait de ce point de vue une inconscience autant qu'une hyperconscience de l'Histoire. Peut-être une partie de l'évolution de la pensée littéraire au $\mathrm{xx}^{\mathrm{e}}$ siècle a-t-elle consisté à dévoiler $a$ posteriori dans l'euphorie des possibles une passion triste, et à mêler progressivement

4. L'expression est de Bergson, Euvres (3e éd.), Paris, PUF, 1959, p. 1340.

5. Ibid.

6. Raymond Queneau, note datée de 1935, Le Voyage en Grèce, Paris, Gallimard, p. 61. 
d'amertume le goût de l'imminence qui faisait la joie de Rivière. Sartre le dit d'une certaine manière en 1939; après avoir, comme on sait, renvoyé dos-à-dos dans La Nausée (1938) le vivre et le raconter, après avoir dévoilé dans le sens de «l'aventure » un irréel, il décrit dans une lettre à Beauvoir l'une de ses lectures romanesques; Sartre y a fait l'expérience de la découverte en soi de ce qui eût été possible, et par conséquent l'aiguillon d'une tristesse douce. Il rapporte en ces termes l'équipée mentale que fut pour lui la lecture de Terre des hommes de Saint-Exupéry, lu en pleine guerre, lorsque sa propre vie se trouve concrètement «barrée » et dépourvue d'horizons pratiques :

Pour une fois je ne regrettais pas ma vie réelle et passée, vous autre, Paris, mon époque, les lieux que j'ai connus. C'était autre chose; beaucoup plus tendre et plus résigné : je regrettais l'Argentine, le Brésil, le Sahara, le monde que je ne connais pas, toute une vie où ni vous ni personne n'aviez de place, une vie que je n'ai pas eue, que j'aurais pu avoir [...]. Je me sentais seul et enfantin, ému comme un tout jeune homme pour un avenir qu'il entrevoit - et en même temps je savais que ça ne serait plus jamais mon avenir; [... je m'étais mis dans la peau du type, c'était formidablement vivant. Et puis c'était une drôle de vie qui n'avait pas cette saveur de mort qu'ont toutes les choses, à présent qu'elles sont barrées par la guerre, une vie toute fraîche, bien au-delà de la guerre et de la paix, parce qu'elle n'existait pas. Je crois bien que c'est la première fois depuis dix ou douze ans que ça m'arrive de rêver à une tout autre vie que la mienne. [...]. Je suis borgne et maladroit, voilà qui suffit à m'écarter pour toujours du métier de pilote de ligne. Mais c'était plutôt une sorte de réalité humaine générale, en moi, qui aurait pu être ça ${ }^{7}$.

Gracq a dit à peu près la même chose, amèrement, décrivant une même déflation : « À vingt ans, à trente ans même, il me semblait que la vie passait très au large et comme insaisissable; une ivresse à arrière-goût d'angoisse se levait de la multiplicité offerte, et massacrée à mesure, des possibles ${ }^{8}$. » L'énergie de la lecture suscite ici une certaine mélancolie, celle de se voir barrer tel ou tel horizon, telle ou telle « ligne de vie», comme l'appelait Thibaudet, alors même que le dispositif narratif fait éprouver au lecteur cette ligne comme une variation en lui de la capacité humaine : cette déclinaison des manières d'être homme lui est ôtée aussitôt qu'offerte.

L'élan d'une multiplication de soi s'est sans doute beaucoup modifié et obscurci dans ce siècle de conflits inattendus, inimaginables, hors

7. Jean-Paul Sartre, Lettres au Castor et à quelques autres, 1926-1963, t. I, éd. Simone de Beauvoir, Paris, Gallimard, 1983, p. 441-442. Je souligne. Je me permets de renvoyer ici à Marielle Macé, «Être ses propres possibles. Lecture et imagination morale chez Sartre», dans Jean-Charles Darmon et Philippe Desan (dir.), Pensée morale et genres littéraires, Paris, PUF, 2009, p. 179-194.

8. Julien Gracq, En lisant en écrivant, Paris, José Corti, p. 78. 
proportions connues, qui fut aussi le siècle de la dévaluation des idées d' " ordre » existentiel, d'histoire orientée, de maîtrise du sens et du réel, bref de destin, et le moment d'une mise en cause de la dignité de la parole. Au sein de ce concert, une voix un peu étrange s'est fait entendre, qui intéresse directement l'énergie d'une possibilisation active de l'individu par la médiation des formes : celle de Paulhan. C'est à l'aventure de Paulhan que je voudrais m'attacher, car elle offre une vision très singulière des formes (en l'occurrence des phrases) que nous pouvons placer à l'horizon de notre action. Le devenir-autre, se-voir-autre, devenir-plus, se joue chez Paulhan dans la logique même de la parole, et offre une issue aux polarités qui nous font opposer trop brutalement le désir d'une autre vie et le risque de l'aliénation, la capacité de s'imaginer autre et la tristesse d'une illusion, l'espérance et les occasions manquées; sa pensée m'invite à ne pas placer la possibilisation de soi dans une confrontation massive des destins réels aux destins imaginaires, mais, à plus petite échelle, dans la dynamique même de l'expression, dans ce qui nous fait nous projeter dans une phrase ou nous construire en elle, car notre parole intérieure est faite en permanence de ces franchissements de la distance de soi à soi, cette distance qui nous sépare intérieurement de nos propres possibles. Lors du long séjour qu'il a fait comme enseignant au lycée de Tananarive à Madagascar, de 1908 à 1910, Paulhan a éprouvé fortement ce que requièrent l'échange verbal et le poids de la parole; il en a conçu une intuition fondamentale sur ce que j'appellerais notre « vie avec les phrases ». On se souvient peut-être du récit de cette troublante expérience de langage, rapportée dans L'Expérience $d u$ proverbe : découvrant l'importance des proverbes dans le dialogue malgache (et, partant, dans le dialogue en général), s'expliquant progressivement leur fonctionnement et la part que lui-même y prend, Paulhan comprend que la tâche de celui qui parle consiste à s'appliquer à seconder ses propres phrases, à « aider» son langage à devenir vrai; non à conformer $a$ priori ce qu'il dit à une sentence-modèle, mais, à l'inverse, à aider les formules proverbiales, une fois qu'il les a avancées, à rejoindre leur circonstance, à trouver au présent leur situation, à accrocher le réel par la force de leurs pinces. Les proverbes suscitent « l'attention, et presque la coopération ${ }^{9}$ » de ceux qui les utilisent, leur effort et leur souci.

Les directions existentielles que dessinent les phrases déjà formées ne sont pas prises ici dans une logique de carrefours qui nous placerait, à chaque étape de la vie, devant un bouquet de destinées potentielles entre lesquelles choisir; c'est un autre mouvement et un autre usage (un autre genre de bovarysme) qui sont mis en lumière, qui me semblent offrir une image particulièrement fine du lien que noue un lecteur de roman avec ce qu'il lit, de la façon dont il figure des pistes à son propre horizon et dont il s'y engage. Paulhan a su laisser fermée " l'armoire aux possibles », mais

9. Jean Paulhan, L'Expérience des proverbes, préface de Jean-Yves Pouilloux, Paris, L'Échoppe, 1993, p. 17. 
c'est pour mieux ouvrir en nous la conscience d'un autre rapport à nos propres capacités, d'un effort particulier de traction de nous-mêmes vers les formes et vers les œuvres.

\section{Les phrases et la poursuite de soi}

Plusieurs textes de Paulhan font allusion, tout en le maintenant à l'état d'énigme, à ce renversement du mouvement expressif consistant à rejoindre une phrase. L'Expérience des proverbes (parmi bien d'autres réflexions sur le fonctionnement de la parole, toutes voisines et convergentes), commencé en 1913 et paru dans Commerce en 1925, nous met sur la voie de cette configuration très singulière, mais qui me semble aussi très générale, du rapport entre un individu et les formes, entre un sujet et les phrasés qui se présentent à lui de l'extérieur. D'abord étonné par le recours permanent aux lieux communs dans le dialogue malgache, se sentant incapable de donner autant que les autres un poids à ses propres phrases, Paulhan comprend progressivement que chaque locuteur emploie son attention et son effort à favoriser l'apparition de ces lieux communs, à les faciliter, et même à recomposer autour d'eux les données de la situation présente, afin de se reconnaître dans des mots partagés; le dialogue « rattrape » les proverbes, comme si les proverbes lancés étaient effectivement en avance, et devenaient la source d'une nouvelle assurance pour celui qui parle.

Dès que le proverbe a été prononcé, il s'agit - étant donné le fait que ce proverbe représente et, exactement, que ce proverbe est - de trouver les phrases qui sont propres à l'exprimer de façon exacte, à le rendre fidèlement. La question est devenue : «Comment ceci voudra-t-il dire le proverbe? $\gg{ }^{10}$.

Il semble ainsi à Paulhan qu'il ait découvert, dans cette observation de l'échange en situation, qu'il nous revient d'aider les mots. D'aider les mots à quoi? À avoir leur force et leur poids, c'est-à-dire à être vrais, ou plutôt à devenir vrais, autrement dit à se « prouver » et à se rejoindre eux-mêmes dans une situation ou une circonstance de vie. L'Expérience du proverbe recèle deux surprises : d'abord la surprise de constater la force des lieux communs, la puissance de traction du langage partagé ${ }^{11}$; puis, dans un second temps - dans cet après-coup si caractéristique de Paulhan - la surprise de comprendre qu'il nous faut les assister dans leur force et pour leur force, coopérer avec leur efficacité, consentir et veiller sur eux, « comme si toutes les choses du monde n'étaient faites que pour être dites ${ }^{12} »$.

10. Ibid., p. 54.

11. C'est ce qu'a montré Laurent Jenny, notamment dans « Terreur et lieu commun », dans Pierre Glaudes (dir.), Terreur et représentation, Grenoble, ELLUG, 2003.

12. Jean Paulhan, Le Don des langues, dans Euvres complètes, t. III, Paris, Cercle du livre précieux, 1966, p. 376. 
Paulhan a exploré inlassablement cette logique de la parole (sans disposer d'une linguistique de l'énonciation, qui lui eût facilité les choses), depuis la publication en 1912, dans le Journal asiatique, des Hain-Teny merinas (ces poèmes malgaches qui sont faits pour la controverse et sont pourtant d'allure toute tendre), jusqu'à celle des Fleurs de Tarbes en 1941. $\mathrm{Au}$ retour de Madagascar, il engage autour de deux projets de doctorat une réflexion plus globale sur les rapports de la parole à la circonstance, et sur la disposition dans laquelle une " phrase d'influence ${ }^{13}$ » trouve celui qui s'apprête à la recevoir ou à la faire sienne. Le même mouvement dialectique (à la fois tellement énigmatique et tellement ordinaire) s'y retrouve. Toute phrase influente, explique-t-il en substance, porte en elle ses possibilités d'animation, et projette autour d'elle un halo de circonstances. Son effet semble « intérieurement et sur le moment, s'inventer et se prouver ${ }^{14}$ ». L'énoncé bien formé crée autour de lui une disposition rétrospective, rayonne en une direction de sens : "Plutôt qu'il ait dû s'adapter aux choses, ce sont les choses qui ont tourné autour de lui »; ce n'est pas lui qui se glisse dans une circonstance mais la circonstance qui le rejoint. De là tout le thème paulhanien du langage comme formation faible, trop suspectée, qu'il faut seconder et aider à rayonner autour d'elle-même.

Les récits de Paulhan, et certaines pages de ses écrits intimes, offrent souvent la même surprise que $L$ 'Expérience des proverbes, celle d'un effort dialectique de la conscience vers le donné des formes, une sorte de chassécroisé temporel où l'on rejoint du « déjà » phrasé, et où l'on est soi-même le champ de la métamorphose - l'espace des possibles. Dans un épisode de sa vie en guerre, Paulhan s'est trouvé non seulement placé devant des phrases à la poursuite desquelles il s'engageait, mais en devoir de susciter lui-même ces formes à rejoindre. Tombé gravement malade en février 1918, envahi par la fièvre, il est pourtant gagné progressivement par un « désir de guérison ». Sa sensibilité aux formes et aux fonctions du langage donne à ce désir (à cette potentialité) une allure toute particulière, et il décide de placer imaginairement devant lui des sortes de slogans :

[...] c'est de la nuit où je me suis mis à répéter, et je ne sais pourquoi j'avais pris ce moment : «Il faut que je veuille guérir, il faut que je veuille guérir... » Alors j'ai renoncé aux images et aux histoires que je me trouvais et j'ai couvert d'inscriptions le mur qui est en face de moi ${ }^{15}$.

Ces inscriptions se fortifient les unes les autres : « Je suis clair », « J'ai mille amis avec moi », « Je suis guéri comme 2 et 2 font 4 »... Leur force d'antécédence, le « déjà » des phrases bien formées, en fait des vérités à rejoindre, où transformer sa propre circonstance. « Du dehors, note Paulhan, bobards

13. Id., La vie est pleine de choses redoutables, Paris, Seghers, 1989, p. 201 et 209.

14. Ibid., p. 199.

15. Ibid., p. 183. 
sur la convalescence. Mais non, bobards excitants [sic] à se créer au-dedans, cet état de joie en avant ${ }^{16}$. " Paulhan a transformé cet épisode en un bref récit, La Guérison sévère; on y retrouve le même cheminement de pensée, la même énergie (exorbitante, sans doute) d'un usage des phrases et d'une progression en soi-même vers elles - comme ces « progrès en amour assez lents » et ces « récits utiles d'amour » dont il a fait une autre nouvelle.

Les phrases disposées au-devant, placées à l'horizon de la conscience d'un individu, lui font ainsi créer à l'intérieur de soi-même un « état de joie en avant », elles lui impriment leur force de directionnalité et d'antécédence. Le projet, ici, est déjà le fait; la phrase vient trouver un interlocuteur qui se trouvait prêt, un individu qui ne suivrait pas tel ou tel mot " s'il n'était en quelque façon embarrassé de lui - et ne le pouvant pas plus retirer que ce pion que l'on vient de placer, au jeu d'échecs, dans une position menaçante à la fois et périlleuse ${ }^{17} \gg$. Dans ce mouvement non seulement celui qui parle est appelé à rejoindre une phrase (au cœur d'un artifice dont il a conscience, dira Paulhan) mais donc aussi à s'y « continuer », à comprendre qu'il ne s'est pas simplement altéré dans une phrase forcée, mais poursuivi lui-même, rejoint dialectiquement et rétrospectivement :

C'est où, disant le proverbe sans rencontrer du premier coup la vive adhésion que plus ou moins confusément j'attendais, je reviens sur mes paroles, je les recommence, je tâche de montrer que «c'était bien ça ». Je me justifie, après coup, ou plutôt je me continue - non pas peut-être sans artifice, ni conscience ${ }^{18}$.

Se poursuivre, se rejoindre, se recommencer, ce sont d'ailleurs les étonnants soulèvements de soi-même qui scandent l'hommage à Rivière que Paulhan donna en 1925-1926.

Ces événements de langage sont d'une couleur très singulière, et très déroutante. Mais ils me semblent correspondre à une sensation commune, celle qu'il nous arrive souvent d'éprouver devant les phrases que l'on lit. Ils peuvent par conséquent nous aider à regarder sous un jour un peu nouveau le va-et-vient entre l'existence et les formes qui survient pendant la lecture - le préfacier des récentes Euvres complètes prétend qu'on peut « devenir fou en lisant Paulhan, amoureux ou bien lecteur »-, ces « sortes d'événements qui regardent tout le monde ${ }^{19} \gg$. J'aimerais en effet mettre au premier plan, en le transposant à l'expérience de la lecture (et pourquoi pas à l'expérience esthétique en général), ce mouvement de soulèvement intérieur du lecteur vers les phrases que lui présentent les livres, ce mouvement qui est une

16. Ibid., p. 185. Je souligne.

17. Ibid., p. 200.

18. Id., L'Expérience du proverbe, op. cit., p. 50.

19. Bernard Baillaud, dans Jean Paulhan, Euvres complètes, I. Récits, Paris, Gallimard, 2006, p. 7. 
forme particulière de possibilisation de soi. Je crois en effet que les phrases que nous lisons sont, à l'intérieur de nous, dans l'attente d'une semblable adéquation, d'un semblable devenirvrai : dans l'attente que nous les fassions advenir non seulement comme sens mais comme intention et comme vérité, que nous les rejoignions à l'intérieur de nous-mêmes, ou que nous les y contestions. Comme les proverbes de Paulhan, les phrases cherchent en nous leur lieu de résonnance; voilà une inversion du rapport d'application de la fiction au réel : les lectures dérivent en nous, disponibles à ce que le réel fasse d'elles un accès; elles ne « s'appliquent » pas au réel pour le recouvrir a posteriori, mais prennent sens en situation. Un aller-et-retour s'instaure dans la lecture entre le donné d'une forme et ce que l'on parvient à en faire dans sa propre vie, en la mettant en phase avec une nouvelle réalité et avec une autre circonstance.

\section{L'avance des formes}

Qu'est-ce qui justifie, au fond, ce mouvement dialectique dont l'énigme a tenu Paulhan toute sa vie, et que j'aimerais placer au cœur de l'acte de lecture? C'est ce que l'on pourrait appeler «l'avance» des formes: la marque de l'antécédence qui touche tout style, son avance perpétuelle sur celui qui l'a risqué (dont il dépasse l'intention), et sur celui qui le reçoit ${ }^{20}$. L'expression est toujours en excès, placée un peu en avant de celui qui parle et, bien sûr, de celui qui écoute. On n' « exprime » pas seulement une intériorité (qui viendrait du dedans, passerait le seuil d'un corps, et trouverait à se dire) dans une phrase, on rejoint quelque chose au dehors; les phrases déjà formées par la littérature peuvent offrir ce dehors; elles désignent nos possibles non parce qu'elles nous exprimeraient, mais justement parce qu'elles nous portent un peu plus loin.

Les livres ont en effet eux aussi « un air d'ancienneté ${ }^{21} »$, cet air d'avoir été là avant nous en venant de quelqu'un d'autre. La précédence, le « déjà » des livres, cette inscription profonde de l'autre - nécessairement ancien - en moi, est à la source d'une émotion particulière dont le roman de Proust, par exemple nous rend souvent familiers : les formes artistiques avec lesquelles Marcel est en lutte ont la force de lui préexister, mais aussi, partant, de le guider, et de le guider en lui-même dès le moment de la lecture; il doit à la fois s'orienter en elles et s'orienter sur elles, «suivre un écrivain dans sa phrase », et prolonger cette phrase en s'incorporant sa force d'avancée. Sa force d'avancée, c'est-à-dire sa capacité « prophétique », comme ne craint pas de le dire Proust : la puissance selon laquelle le livre a risqué une forme, et par là proposé une direction. Le moment esthétique est l'ouverture de ce

20. Voir à ce sujet, dans le sillage de la pensée de l'expression élaborée toute sa vie durant par Merleau-Ponty, La Parole singulière de Laurent Jenny (Paris, Belin, 1991, rééd. 2009).

21. L'expression est, à un tout autre propos, de Jean-Christophe Bailly dans Le Versant animal, Paris, Bayard, 2007. 
décalage qui pourra trouver plus tard son emploi. Le sentiment de prophétie n'émousse pas la nouveauté de ce qui est à vivre, il est simplement l'écho affectif, le retentissement intérieur de cette longueur d'avance que les phrases littéraires ont par définition sur nous, de leur excès dont les circonstances de la vie nous permettront ou pas de « rattraper » l'énergie et la novation.

C'est pourquoi je comparerais volontiers l'expérience de lecture à cette énergie de la phrase paulhanienne, phrase à transformations, « tournée en chose, à la fois simplifiée et prête à être appliquée ${ }^{22}$ », qui tire de la nécessité même de procéder à ces transformations le plus clair de son influence. Ce que je lis, en effet, n'est pas ce qui m'indique une conduite à imiter, un destin dans lequel me projeter, comme si je me confiais à une tout autre existentialité, mais ce qui me ressemble « déjà » en se plaçant au-devant de moi, ce qui m'appelle un peu au-delà de moi-même, exige ma coopération, requérant de moi un effort contre moi. Il ne s'agit pas exactement de l'espoir d'être précédé par un idéal, du bonheur d'être conduit par un type, de l'illusion butée d'un bovarysme des sentences, mais de se reconnaître juste à côté ${ }^{23}$. Car ces phrases sont des continuations de soi placées au-devant, autrement dit un soi déjà possible; l'acte de lire nous les fait éprouver comme telles, et immédiatement. En cela, la pulsion de reconnaissance de soi dans la lecture est aussi une reconnaissance par anticipation : cette singularité dans quoi je me retrouve, peut-être suis-je en train de l'inventer; il faudra que je la rejoigne, et que je m'y rejoigne; c'est moi hors de moi - parce que j'y suis déjà et que je m'y reconnais, parce que je vais le devenir, ou simplement parce que je veux le devenir. Le possible, celui qui surgit dans la lecture comme l'image d'une vie pour le lecteur, est peut-être cela : la représentation d'un avant que l'on se donne sur-le-champ pour s'y rejoindre, pour y rejoindre sa propre puissance d'être. Le goût du possible n'y est pas l'identification du « soi » à une destinée autre (l'aliénation, l'oubli du présent, l'abandon à une identification butée ou une multiplicité illusoire), mais une sorte de soulèvement de soi par soi, de différenciation de soi-même placée en avant de soi-même, de projection de soi dans l'appropriation rétrospective de mots communs et de cadres nouveaux. Il suppose un va-et-vient permanent entre le donné (la phrase proposée, qui par définition emporte un peu plus loin et anticipe ce qu'elle est déjà) et ce qui est à inventer; ce qui est en avant de moi est tout ensemble passé et futur, déjà vu et estrangement. Il ne dépend que de moi de le faire, comme le proverbe, « exact, précis, encourageant ${ }^{24}$ ».

Les phrases de romans, comme celles de Paulhan, viennent d'avant et d'autrui, mais le lecteur les place donc devant lui, à l'horizon actif de sa propre parole et de ce qu'il a à vivre. Le mouvement permanent de la lecture

22. Id., La vie est pleine de choses redoutables, op. cit., p. 214.

23. L'expression est de Philippe Jousset, Anthropologie du style. Propositions, Pessac, Presses universitaires de Bordeaux, 2008.

24. Jean Paulhan, La vie est pleine de choses redoutables, op. cit., p. 206. 
me paraît être cet effort dialectique de poursuite de soi et d'altération de soi que nous effectuons à chaque instant avec les phrases, effort vers les mots que la lecture, comme simple acte d'actualisation linguistique et mentale, a déjà dû réaliser en nous : étonnés devant ces phrases comme devant notre propre parole, ce que nous avançons nous dépasse, nous devance, et nous devons le rejoindre après coup (c'est dans ce mouvement de poursuite que l'éthique de la parole de Paulhan se dissocie des surprises reconnues par le surréalisme ou la psychanalyse). La considération des « possibles » ouverts par la rencontre avec une fiction s'en trouve peut-être déplacée, car la nature même de la phrase est ici essentielle : « La littérature est faite de phrases qui se donnent pour ce qu'elles sont. La fiction montre en toute clarté comment les phrases, en disant quelque chose, font quelque chose ${ }^{25}$. » Les possibles auxquels la lecture romanesque nous disposent ne sont pas forcément des conduites, des choix de contenus, des descriptions de destinées, de caractères ou de modalités d'action; mais, souvent, des allures phrastiques, ce que Barthes appellera des « airs syntaxiques ». Sartre par exemple avait sa phrase préférée (d'ailleurs elle est fausse) extraite du Rouge et le noir : « Tant qu'il put voir le clocher de Verrières, souvent Julien se retourna... »; cette phrase lui donnait l'impulsion régulière d'une simple attitude temporelle et le désir de faire, comme il le dit dans ses Carnets de guerre, « un petit tour dans l'avenir » afin de se retourner sur son jeune destin et de hocher la tête...

La dimension de la phrase, en fait, est l'échelle-même de notre attention dans la lecture; ce n'est pas seulement une fois le livre refermé que nous revenons à nous-mêmes, c'est phrase après phrase que nous acquiesçons ou protestons, que nous nous projetons dans une situation de langage ou que nous nous en détachons, que nous réglons la distance avec le livre, bref, que nous nous orientons (grâce au défilé des formes extérieures) dans nos propres potentialités. Soulignons cette immédiateté de la remobilisation, l'immédiateté de l'évaluation vitale du sens et de l'application de ce sens à soi, c'est-à-dire l'élargissement à toute la lecture de cette « refiguration » que Ricœur ne plaçait qu'au terme du processus mimétique. La possibilisation de soi n'est pas seulement l'aboutissement postérieur, mais le moteur continu de la lecture, des décisions qui l'animent pas à pas, de sa dialectique finement rythmée.

Le lecteur consent donc lui aussi à un effort (à un « artifice ») pour « rattraper » les formes, poursuivre, accrocher les phrases lues et les accommoder; l'effort attentionnel et la tentative pour redisposer en soi les conditions de l'expérience quand on lit est déjà une transformation de soi; cependant, peut-être le lecteur sent-il encore que, dans cet effort, il ne s'est pas porté ailleurs mais il s'est poursuivi lui-même. Les phrases lues sont non seulement des pistes à suivre, mais aussi des voies où se rejoindre soi-même, où « se continuer », comme le dit si bien Paulhan (« Je me justifie, après coup, ou plutôt je me continue »); autrement dit des possibles sur-le-champ. 
Ici encore c'est selon une phrase que l'on se « possibilise » tout particulièrement. Car une phrase est non pas descriptive mais « instauratrice ${ }^{26}$ », elle suscite en elle-même sa propre origine, façonne son propre passé; lorsque nous en sommes les lecteurs, cette qualité singulière de passé s'installe en nous, elle nous prête son orientation, nous lui prêtons notre durée intérieure, et la lecture l'effectue comme nôtre. La lecture ne nous fait pas répondre à l'indétermination (remplir des lacunes) mais au contraire à la densité des formes, à ce « trop dit » des livres qui est l'énergie à laquelle nous nous guidons nousmêmes. C'est alors que l'on transforme ce que l'on lit en voie perceptive, sémantique, ou pragmatique; que l'on retrouve la piste qui l'a suscitée et en a fait une trace, que l'on relance cette décision expressive de l'auteur en même temps qu'on la suit.

Paulhan aurait sans doute montré la même ironie que Bergson devant l'illusion qu'une sorte d' " armoire aux possibles » est disposée à côté de nous, comme un réservoir ou une bombe, et qu'il nous suffirait d'en posséder magiquement la clé pour imaginer l'altérité de qui s'apprête à surgir. Comme si les possibles étaient ces directions concurrentes en attente d'actualisation, entre lesquelles le wagon-lecteur se déplacerait au gré des aiguillages narratifs, comme si le roman même était fait pour nous donner avant tout ce sentiment-là, ce sentiment d'une imminence à portée de main. Son idée de notre propre avenir, et de notre rapport aux formes, n'était pas celle-ci, car il était sensible plus qu'aucun autre à la force de l'antécédence et de la rétrospection, à la pression en nous-mêmes de l'advenu et du commun, à l'idée que toute « première fois » survient comme une « deuxième ». Le prospectif-rétrospectif qu'il a mis en valeur, sans tout à fait l'élucider, me semble allégoriser la pulsation même de la lecture, ce rythme long qui fait que l'on ne se projette pas forcément dans un destin totalement autre une fois le livre refermé, mais qu'on aura fait l'expérience de toutes sortes de bifurcations intérieures dans la régulation continue et l'abréviation d'une distance, celle de notre rapport aux phrases littéraires et à cette « longueur d'avance » qui les définit. Paulhan m'aide à comprendre que la littérature ne nous indique pas exactement des voies potentielles à prendre, mais nous conduit plutôt à nous orienter à l'intérieur de nos propres possibles, c'est-à-dire à nous poursuivre en nous altérant. Ici, rattraper les phrases n'est pas abandonner aux livres la charge d'un « devenir soi-même », c'est s'appliquer à s'y reconnaître, se continuer, s'étonner devant ce dont on aura donc été capable. C'est la beauté ordinaire d'un mouvement tournant de l'individuation, dont on fait l'expérience dans les moments esthétiques.

Marielle Macé

CRAL, CNRS-EHESS 